\title{
Correction to: Results on the Robust Observer-Based Position Controller for Parallel Kinematic Machines
}

\author{
Margareta Stefanovic $^{1} \cdot$ Huijuan Zhang $^{2}$
}

Published online: 5 January 2018

(c) Springer Science+Business Media B.V. 2017

Correction to: J Intell Robot Syst (2012) 66:417-428

https://doi.org/10.1007/s10846-011-9622-0

A correction is being made regarding Assumption 2: the assumption does not hold for the system under consideration, where only displacement measurements are available, but may hold under weaker conditions that allow partial velocities availability as well.

We thank Prof. Brian Driessen for bringing this matter to our attention.

In addition, both authors have updated their affiliations. The new affiliations are given in this article.

The online version of the original article can be found at https://doi.org/10.1007/s10846-011-9622-0.

Margareta Stefanovic

mstefan8@du.edu

Huijuan Zhang

hzhang@safoco.com

1 Department of Electrical and Computer Engineering,

University of Denver, Denver, CO, USA

2 Safoco Inc., 11421-A Todd St, Houston, TX 77055, USA 